\title{
Damping of Torsional Beam Vibrations by Control of Warping Displacement
}

\author{
Høgsberg, Jan Becker; Hoffmeyer, David; Ejlersen, Christian
}

Published in:

Journal of Vibration and Acoustics

Link to article, DOI:

$10.1115 / 1.4031616$

Publication date:

2016

Document Version

Peer reviewed version

Link back to DTU Orbit

Citation (APA):

Høgsberg, J. B., Hoffmeyer, D., \& Ejlersen, C. (2016). Damping of Torsional Beam Vibrations by Control of Warping Displacement. Journal of Vibration and Acoustics, 138, [014501]. https://doi.org/10.1115/1.4031616

\section{General rights}

Copyright and moral rights for the publications made accessible in the public portal are retained by the authors and/or other copyright owners and it is a condition of accessing publications that users recognise and abide by the legal requirements associated with these rights.

- Users may download and print one copy of any publication from the public portal for the purpose of private study or research.

- You may not further distribute the material or use it for any profit-making activity or commercial gain

- You may freely distribute the URL identifying the publication in the public portal

If you believe that this document breaches copyright please contact us providing details, and we will remove access to the work immediately and investigate your claim. 


\title{
DAMPING OF TORSIONAL BEAM VIBRATIONS \\ BY CONTROL OF WARPING DISPLACEMENT
}

\author{
Jan Høgsberg $^{1 *}$, David Hoffmeyer ${ }^{1,2}$ And Christian EJlersen $^{1,2}$ \\ ${ }^{1}$ Department of Mechanical Engineering, \\ Technical University of Denmark, DK-2800, Kongens Lyngby, Denmark \\ ${ }^{2}$ Rambøll, Hannemanns Alle 53, DK-2300, Copenhagen, Denmark
}

\begin{abstract}
Supplemental damping of torsional beam vibrations is considered by viscous bimoments acting on the axial warping displacement at the beam supports. The concept is illustrated by solving the governing eigenvalue problem for various support configurations with the applied bimoments represented as viscous boundary conditions. It is demonstrated that properly calibrated viscous bimoments introduce a significant level of supplemental damping to the targeted vibration mode and that the attainable damping can be accurately estimated from the two undamped problems associated with vanishing and infinite viscous parameters, respectively.
\end{abstract}

\section{INTRODUCTION}

For thin-walled beams the out-of-plane warping displacement associated with inhomogeneous torsion is often significant at the boundaries of beams with open cross-sections. Thus, for these types of thin-walled beams the restraining of warping may result in an often appreciable increase in natural frequency and change in vibration characteristics. The analysis of torsional vibrations of beams, including the effect of restrained warping, dates back to Gere [1], who solved the governing equation by separation of variables and presented results for wave numbers and natural frequencies for typical boundary conditions. The coupling with bending was afterwards considered in Gere and Lin [2] and in Lin [3] for beams restrained against lateral displacements. For the uncoupled problem Zhang and Chen [4] derived the mass and stiffness matrices from the exact dynamic interpolation functions, while Eisenberger [5,6] considered torsional vibrations of a beam with varying cross-section properties. The influence of elastic support conditions has been considered by Christiano and Salmela [7]. They demonstrated that elastic boundaries can significantly modify the torsional vibration characteristics because restraining of warping is a localized effect at the supports of the beam. In the present brief this localized effect is specifically utilized to introduce substantial supplemental damping by applying viscous boundary conditions through supporting bimoments. Damping of torsional vibrations via the out-of-plane warping deformation has been investigated by Narayanan et al. [8,9], who considered viscoelastic layers attached to the flanges

\footnotetext{
${ }^{*}$ Corresponding author: jhg@mek.dtu.dk
} 
of a thin-walled beam. The present brief instead concerns the application of external viscous bimoments acting locally at the supports. The eigenvalue problem associated with free torsional vibrations are solved with respect to the complex-valued natural frequency and the corresponding damping ratio. It is demonstrated that substantial damping ratios can be realized by viscous bimoments, which indicates the potential of the present approach.

\section{Free torsional Vibrations}

Consider torsional vibrations of a beam with length $\ell$, longitudinal axis $x$ and transverse axes $\{y, z\}$, see Fig. 1a. The axial displacement is $u(x, y, z, t)$ and the angle of twist is $\theta(x, t)$, where $t$ denotes time. Figure $1 \mathrm{~b}$ illustrates the axial warping displacement $u(x, y, z, t)$ for a thin-walled I-section, and this displacement is written in separated form as $u=-\psi \theta^{\prime}$, where the negative rate of twist $-\theta(x, t)$ and sectorial coordinate $\psi(y, z)$ represent the intensity and shape of out-of-plane warping, respectively. The notation ()$^{\prime}=\partial() / \partial x$ and ()$=\partial() / \partial t$ is adopted in the following.

2.1. Equilibrium equation. For constant cross-section parameters the partial differential equation governing torsional vibrations can be written as $[1,2]$

$$
E I \theta^{\prime \prime \prime \prime}-G K \theta^{\prime \prime}+\rho J \ddot{\theta}=0
$$

where $G K$ is the stiffness associated with homogeneous (St. Venant) torsion, EI is the corresponding warping stiffness for inhomogeneous (Vlasov) torsion, while $\rho J$ represents the torsional inertia. The boundary conditions for this fourth order differential equation are expressed in terms of the angle of twist $\theta(x, t)$, the warping intensity $-\theta^{\prime}(x, t)$, the bimoment $B(x, t)=-E I \theta^{\prime \prime}(x, t)$ and the torsional moment $M(x, t)=G K \theta^{\prime}(x, t)-E I \theta^{\prime \prime \prime}(x, t)$.

2.2. Free vibration solution. Free vibrations are investigated by assuming a time harmonic solution $\theta=\varphi \mathrm{e}^{i \omega t}$, where $\omega$ is the angular frequency and $\varphi(x)$ is the spatial solution. Substitution into (1) gives the ordinary differential equation

$$
\varphi_{j}^{\prime \prime \prime \prime}-k^{2} \varphi_{j}^{\prime \prime}-\lambda_{j}^{4} \varphi_{j}=0
$$

with the warping length scale $k$, wave speed $c$ and wave number $\lambda$ defined as

$$
k^{2}=\frac{G K}{E I} \quad, \quad c^{2}=\frac{G K}{\rho J} \quad, \quad \lambda^{4}=\left(\frac{k \omega}{c}\right)^{2}
$$

The homogeneous equation (2) forms together with homogeneous boundary conditions an eigenvalue problem with eigenvalue $\lambda$ or $\omega$ and eigenfunction $\varphi(x)$. Following Gere [1] the solution to (2) gives the vibration form $\varphi(x)$ in terms of hyperbolic and trigonometric functions

$$
\varphi=D_{1} \cosh (\alpha x)+D_{2} \sinh (\alpha x)+D_{3} \cos (\beta x)+D_{4} \sin (\beta x)
$$

introducing four arbitrary constants $D_{1}$ to $D_{4}$ and two apparent wave numbers

$$
\alpha^{2}=\sqrt{\frac{1}{4} k^{4}+\lambda^{4}}+\frac{1}{2} k^{2} \quad, \quad \beta^{2}=\sqrt{\frac{1}{4} k^{4}+\lambda^{4}}-\frac{1}{2} k^{2}
$$


The two wave numbers $\alpha$ and $\beta$ satisfy the relations

$$
\alpha^{2}=\beta^{2}+k^{2} \quad, \quad \alpha \beta=\lambda^{2}
$$

where the former gives $\alpha$ with respect to $\beta$, while the latter together with (3c) determines $\omega$.

2.3. Viscous boundary condition. The boundary damping concept is illustrated in Fig. 2 for a beam with two 'simple' supports, which prevent twist but allow warping. At the right support a normal stress distribution $\sigma_{d}(y, z, t)$ reacts on the axial displacement $u$, producing a bimoment $B(\ell, t)=B_{d}(t)$ at the support.

Damping is introduced by assuming a viscous stress $\sigma_{d}(y, z, t)$ proportional to the collocated axial velocity $\dot{u}_{d}(y, z, t)$. The viscous gain parameter is $g$ and the normal stress at the boundary is

$$
\sigma_{d}= \pm g E \dot{u}_{d}
$$

The stress is defined positive in tension, while $\dot{u}_{d}(y, z, t)$ is positive in the $x$-direction. Thus, for the normal stress to be reactive the ' + ' in (7) refers to the left boundary at $x=0$, while the ' - ' is used at $x=\ell$. The axial velocity at the boundaries $\dot{u}_{d}(y, z, t)$ is also written in separated form,

$$
\dot{u}_{d}=-h \psi \dot{\theta}_{d}^{\prime}
$$

where $h(y, z)$ represents the distribution of the viscous damping treatment over the cross section, while $-\dot{\theta}_{d}^{\prime}(t)$ is the time derivative of the warping intensity.

The normal stress distribution $\sigma_{d}(y, z, t)$ produces a resulting bimoment $B_{d}(t)$ at the damped boundaries,

$$
B_{d}=\mp g E I_{d} \dot{\theta}_{d}^{\prime}
$$

where the warping stiffness from the viscous stress distribution is defined as

$$
E I_{d}=\int_{A} E(h \psi)^{2} d A
$$

with $A$ representing the cross section area. The applied bimoment $B_{d}(t)$ must be balanced at the boundary by the section bimoment $B(x, t)=-E I \theta(x, t)^{\prime \prime}$. This yields the damping boundary condition

$$
\theta_{d}^{\prime \prime} \mp \eta \dot{\theta}_{d}^{\prime}=0
$$

where ' - ' is used in $x=0$ and ' + ' in $x=\ell$. The effective viscous damping parameter is introduced as $\eta=g E I_{d} / E I$.

\section{Damping of torsional BeAm Vibrations}

The potential of imposing viscous bimoments at the boundaries to damp torsional beam vibrations is demonstrated for the non-dimensional length scales $k \ell=3$ and 30 , representative of a slender beam with open and closed cross section, respectively. First damping of a beam with symmetric 'simple-simple' boundary conditions is investigated, while non-symmetric 'simple-free' supports are considered next. With respect to boundary conditions 'free' refers to free twist and free warping, 
while 'simple' prevents twist and 'fixed' prevents both twist and warping. A 'free' support with restrained warping is referred to as 'no warping'. In the following solutions are presented for the first vibration mode.

3.1. Damping of 'simple-simple' beam. The viscous boundary condition (11) is introduced in three subsequent steps, as illustrated by cases (I)-(III) in Fig. 3. In (I) the beam has 'simple-simple' supports and the viscous bimoment (indicated by the double arrow in the figure) is applied the left boundary in $x=0$. As $\eta \rightarrow \infty$ warping is restrained and the supports become 'fixed-simple'. For this 'fixed-simple' case (II) the viscous bimoment is then applied at the 'simple' support in $x=\ell$ and as $\eta \rightarrow \infty$ 'fixed-fixed' support conditions are finally reached. Thus, the sequence (I) to (II) constitutes the transition from a 'simple-simple' beam to a 'fixed-fixed' beam by sequentially applying viscous damping at the two ends. Case (III) is the combined effort with viscous damping applied simultaneously at the two supports.

The four boundary conditions for each of the cases (I)-(III) are summarized in the upper part of Table 1. Solving (4) by the four homogeneous boundary conditions yields the transcendental equation presented in the table and a solution $\varphi(x)$ for the corresponding vibration form. The individual transcendental equations are solved with respect to $\beta \ell$ with $\alpha \ell$ determined by (6a), while the natural frequency $\omega$ follows from (3c) with the wave number $\lambda$ given in (6b). For finite values of the damping parameter $\eta$ the transcendental equations are solved to obtain the complex-valued natural frequency $\omega$ and the corresponding damping ratio $\zeta=\operatorname{Im}[\omega] /|\omega|$.

For $\eta=0$ the transcendental equations for cases (I)-(III) in Table 1 govern the undamped solutions for 'simple-simple' and 'simple-fixed' beams, while for $\eta \rightarrow \infty$ associated with restrained warping they determine the undamped solutions for 'simple-fixed' and 'fixed-fixed' supports. These undamped vibration problems associated with cases (I)-(III) have also been solved in [1] and the natural frequencies are represented by the three upper curves in Fig. 4. The dashed vertical lines in the figure identify the solutions for $k \ell=3$ and 30 and as in [1] the curves are normalized by the natural frequency $\omega_{s s}$ for 'simple-simple' supports.

Figure 5 shows the frequency loci $(\mathrm{a}, \mathrm{c})$ and damping ratio (b,d) for the three cases (I)-(III). The individual loci initiate and terminate at the undamped natural frequencies $\omega_{0}$ and $\omega_{\infty}$ associated with $\eta=0$ and $\eta \rightarrow \infty$, respectively. For the individual cases $\omega_{\infty}>\omega_{0}$ because the restraining of warping implies an increase in natural frequency. It is seen that the damping contribution from cases (I) and (II) is practically identical with $\zeta_{\max }^{(\mathrm{I})} \simeq \zeta_{\max }^{(\mathrm{II})}=0.18$ for $k \ell=3$ in Fig. 5 (b) and 0.017 for $k \ell=30$ in Fig. $5(\mathrm{~d})$. The maximum damping is approximately doubled in the combined case (III) to $\zeta_{\max }^{(\mathrm{III})}=0.41$ for $k \ell=3$ and 0.036 for $k \ell=30$.

In Fig. 5(a,c) the trajectories of $\omega$ are practically semi-circular and the maximum attainable damping can therefore be estimated by the relative radius as $\zeta_{\max } \simeq \frac{1}{2}\left(\omega_{\infty}-\omega_{0}\right) / \omega_{0}$. With the limiting frequencies $\omega_{0}$ and $\omega_{\infty}$ represented by the undamped solutions in Fig. 4 the attainable 
damping can be estimated without solving the complex eigenvalue problem. In case (I) the relative increase in frequency from 'simple-simple' (representing $\omega_{0}$ ) to 'simple-fixed' (representing $\omega_{\infty}$ ) is $\left(\omega_{\infty}-\omega_{0}\right) / \omega_{0}=0.39$ for $k \ell=3$, while in case (III) from 'simple-simple' $\left(\omega_{0}\right)$ to 'fixed-fixed' $\left(\omega_{\infty}\right)$ it is 0.81 . These estimates agree well with $2 \zeta_{\max }$ obtained from Fig. $5(\mathrm{~b})$, which demonstrates that the attainable damping level can be accurately predicted by the undamped support conditions associated with $\eta=0$ and $\eta \rightarrow \infty$.

3.2. Damping of 'simple-free' beam. The damping of a beam with non-symmetric 'simplefree' supports is now considered. As shown in Fig. 3 this problem is also divided into two sequential cases (IV)-(V) and a final case (VI) with combined damping at both boundaries. For $\eta=0$ the cases (IV)-(VI) represent 'simple-free' and 'fixed-free', while for $\eta \rightarrow \infty$ they recover 'fixed-free' and 'fixed-no warping' supports. These undamped solutions associated with $\eta=0$ and $\eta \rightarrow \infty$ are represented by the bottom three curves in Fig. 4. The boundary conditions and corresponding transcendental equations are summarized in the bottom half of Table 1 and the root loci and damping ratios are shown in Fig. 6.

It is seen that the root locus of case (IV) is significantly larger than for case (V), which practically vanishes for $k \ell=30$. This difference is also observed in Fig. 4 for $k \ell=3$, where the frequency increase $\left(\omega_{\infty}-\omega_{0}\right) / \omega_{0}=0.39$ for case (IV) from 'simple-free' to 'fixed-free' is larger than the increase $\left(\omega_{\infty}-\omega_{0}\right) / \omega_{0}=0.10$ for case $(V)$ from 'fixed-free' to 'fixed-no warping'. For the combined case (VI) from 'simple-free' to 'fixed-no warping' the relative increase is $\left(\omega_{\infty}-\omega_{0}\right) / \omega_{0}=0.54$. Thus, applying the viscous bimoment at the left support constitutes the main contribution to the combined damping case. The damping ratios obtained from the complex-valued natural frequencies are shown in Fig. 6(b,d). For $k \ell=3$ the actual case (IV) damping ratio in Fig. 6 (b) is $\zeta_{\max }^{(\mathrm{IV})}=0.20$, while it is only slightly larger in combined case (VI) with $\zeta_{\max }^{(\mathrm{VI})}=0.27$. For $k \ell=30$ the attainable damping ratio in Fig. $6(\mathrm{~d})$ is $\zeta_{\max }^{(\mathrm{IV})} \simeq \zeta_{\max }^{(\mathrm{VI})}=0.017$. Thus, the outcome of applying damping at the right 'free' end is only limited.

In the final case (VI) viscous damping is applied at both supports of the 'simple-free' beam, see Fig. 3. However, the supports are not identical and the two viscous gains should therefore not be equal. Thus, $\eta$ represents the gain at the left support in $x=0$, while $\nu \eta$ is the gain at $x=\ell$, whereby $\nu$ represents the ratio between the applied viscous gains. An optimal balance between the viscous bimoments is determined by their individual ability to restrain warping for increasing gain. As demonstrated in [10] this balance condition can be formulated as

$$
\frac{B_{d}^{0}(\ell)}{B_{d}^{0}(0)}=\frac{B^{\infty}(\ell)}{B^{\infty}(0)}
$$

where $B_{d}^{0}$ is the bimoment produced at the viscous support for the undamped vibration mode, while $B^{\infty}$ is the reaction bimoment for restrained warping associated with $\eta \rightarrow \infty$. When $\varphi_{0}(x)$ represents the vibration form with 'simple-free' supports and $\varphi_{\infty}(x)$ is for 'fixed-no warping' 
supports, substitution into (12) gives the optimal gain ratio

$$
\nu=-\frac{\varphi_{0}^{\prime}(0) \varphi_{\infty}^{\prime \prime}(\ell)}{\varphi_{0}^{\prime}(\ell) \varphi_{\infty}^{\prime \prime}(0)}
$$

For $k \ell=3$ and 30 this expression gives $\nu=0.89$ and 1.05 , respectively. To verify the accuracy of (13) the transcendental equation (VI) in Table 1 is solved for all combinations of $\nu$ and $\eta$, and the maximum damping ratio $\zeta_{\max }$ is determined by a numerical search procedure. The maximum damping ratio is shown in Fig. 7 for $k \ell=3$. The dashed line represents $\nu=0.89$ and it is seen to correspond well with the maximum of the curve. A more detailed description of the balancing of viscous dampers can be found in [10]. The case (VI) solution curves in Fig. 6(a,b) and (c,d) are determined for $\nu=0.89$ and 1.05 , respectively.

\section{Discussion}

The present concept proposes to apply a viscous bimoment at the beam boundaries to introduce damping to the torsional vibration modes of a beam. It has been demonstrated that substantial damping can potentially be realized for open cross-sections with small non-dimensional length scales $k \ell$, while for increasing $k \ell$ the attainable damping is reduced. Because the supplemental damping is introduced through bimoments as viscous boundary conditions it is shown how the attainable damping can be accurately estimated from the relative difference in natural frequency between the two undamped cases associated with free and restrained warping at the damped boundary.

The theoretical results illustrate the effectiveness of applying viscous bimoments and thereby introduce the warping control concept as a potential candidate for damping of torsional vibrations. This of course assumes that the desired bimoment can be realized locally at the supports. And since the axial warping displacements $u_{d}$ are typically small, active control concepts are probably more feasible than passive means. A potential device is the piezoelectric stack transducer and load cell placed in series and controlled by Integral Force Feedback (IFF). This concept provides large forces at small stroke levels and has effectively been used in [11] for damping of truss structures and is described in detail in [12]. When placing four of these local devices in a sufficiently accurate and self-equilibrating configuration on the specific beam cross-section the IFF control should be effective in introducing the desired viscous bimoment to damp torsional vibrations.

\section{REFERENCES}

[1] J.M. Gere, 1954. Torsional vibrations of beams of thin-walled open section, Journal of Applied Mechanics, 21:381-387.

[2] J.M. Gere and Y.K. Lin, 1958. Coupled vibration of thin-walled beams of open cross section, Journal of Applied Mechanics, 25:373-378.

[3] Y.K. Lin, 1960. Coupled vibrations of restrained thin-walled beams, Journal of Applied Mechanics, 82:373-378. 
[4] Z. Zhang and S. Chen, 1991. A new method for the vibration of thin-walled beams, Computers and Structures, 39:597-601.

[5] M. Eisenberger, 1995. Nonuniform torsional analysis of variable and open cross-section bars, Thin-Walled Structures, 21:93-105.

[6] M. Eisenberger, 1997. Torsional vibrations of open and variable cross-section bars, Thin-Walled Structures, 28:269-278.

[7] P. Christiano and L. Salmela, 1971. Frequencies of beams with elastic warping restraint, Journal of the Structutal Division, 97:1835-1840.

[8] S. Narayanan, J.P. Verma and A.K. Mallik, 1981. Free vibration of thin-walled open section beans with unconstrained damping treatment, Journal of Applied Mechanics, 48:169-173.

[9] S. Narayanan and A.K. Mallik, 1981. Free vibration of thin walled open section beans with constrained damping treatment, Journal of Sound and Vibration, 74:429-439.

[10] J.A. Main and S. Krenk, 2005. Efficiency and tuning of viscous dampers on discrete systems, Journal of Sound and Vibration, 286:97-122.

[11] A. Preumont, J-.P. Dufour and C. Maleékian, 1992. Active damping by a local force feedback with piezoelectric actuators, Journal of Guidance, Control and Dynamics, 15:390-395.

[12] A. Preumont. 2011. Vibration Control of Active Structures. An Introduction, 3rd edition, Springer, Heidelberg.

\section{List OF FIGURES}

1 (a) Coordinates and degrees of freedom, (b) warping displacement of beam.

2 Beam with simple supports and reaction stress $\sigma_{d}$ due to warping displacement at $x=\ell . \quad 10$

3 Cases (I)-(VI) with a double arrow representing a viscous bimoment.

4 Undamped natural frequencies for the first vibration mode.

5 Complex frequency loci (a,c) and damping ratios (b,d) for $k \ell=3(\mathrm{a}, \mathrm{b})$ and $k \ell=30(\mathrm{c}, \mathrm{d}) .13$

6 Frequency loci $(\mathrm{a}, \mathrm{c})$ and damping ratios $(\mathrm{b}, \mathrm{d})$ for $k \ell=3(\mathrm{a}, \mathrm{b})$ and $k \ell=30(\mathrm{c}, \mathrm{d})$.

7 Maximum damping ratio as function of viscous ratio $\nu$ for $k \ell=3$.

\section{List OF TABLES}

1 Boundary conditions and transcendental equations for cases (I) to (VI). 
TABLE 1. Boundary conditions and transcendental equations for cases (I) to (VI).

(I)

$$
\varphi(0)=0, \quad \varphi^{\prime \prime}(0)-i \omega \eta \varphi^{\prime}(0)=0, \quad \varphi(\ell)=0, \quad \varphi^{\prime \prime}(\ell)=0
$$

$\left((\alpha \ell)^{2}+(\beta \ell)^{2}\right) \sinh (\alpha \ell) \sin (\beta \ell)-i \omega \eta \ell \cosh (\alpha \ell) \cos (\beta \ell)[\beta \ell \tanh (\alpha \ell)-\alpha \ell \tan (\beta \ell)]=0$

(II)

$$
\varphi(0)=0, \quad \varphi^{\prime}(0)=0, \quad \varphi(\ell)=0, \quad \varphi^{\prime \prime}(\ell)+i \omega \eta \varphi^{\prime}(\ell)=0
$$

$$
\left((\alpha \ell)^{2}+(\beta \ell)^{2}\right) \cosh (\alpha \ell) \cos (\beta \ell)[\beta \ell \tanh (\alpha \ell)-\alpha \ell \tan (\beta \ell)]
$$

$-i \omega \eta \ell\left[\left((\alpha \ell)^{2}-(\beta \ell)^{2}\right) \sinh (\alpha \ell) \sin (\beta \ell)+2(\alpha \ell)(\beta \ell)(1-\cosh (\alpha \ell) \cos (\beta \ell))\right]=0$

(III)

$$
\varphi(0)=0, \quad \varphi^{\prime \prime}(0)-i \omega \eta \varphi^{\prime}(0)=0, \quad \varphi(\ell)=0, \quad \varphi^{\prime \prime}(\ell)+i \omega \eta \varphi^{\prime}(\ell)=0
$$

$$
\frac{(\alpha \ell)^{2}+(\beta \ell)^{2}}{i \omega \eta \ell} \sinh (\alpha \ell) \sin (\beta \ell)-2 \cosh (\alpha \ell) \cos (\beta \ell)[\beta \ell \tanh (\alpha \ell)-\alpha \ell \tan (\beta \ell)]
$$

$+\frac{i \omega \eta \ell}{(\alpha \ell)^{2}+(\beta \ell)^{2}}\left[\left((\alpha \ell)^{2}-(\beta \ell)^{2}\right) \sinh (\alpha \ell) \sin (\beta \ell)+2(\alpha \ell)(\beta \ell)(1-\cosh (\alpha \ell) \cos (\beta \ell))\right]=0$

(IV)

V)

$$
\begin{aligned}
& \varphi(0)=0, \quad \varphi^{\prime \prime}(0)-i \omega \eta \varphi^{\prime}(0)=0, \quad \varphi^{\prime \prime}(\ell)=0, \quad k^{2} \varphi^{\prime}(\ell)-\varphi^{\prime \prime \prime}(\ell)=0 \\
& \frac{(\alpha \ell)^{2}+(\beta \ell)^{2}}{(\alpha \ell)^{2}(\beta \ell)^{2}} \cosh (\alpha \ell) \cos (\beta \ell)\left[(\alpha \ell)^{3} \tanh (\alpha \ell)-(\beta \ell)^{3} \tan (\beta \ell)\right] \\
& +i \omega \eta \ell\left[\frac{(\alpha \ell)^{4}+(\beta \ell)^{4}}{(\alpha \ell)^{2}(\beta \ell)^{2}} \cosh (\alpha \ell) \cos (\beta \ell)+\frac{(\alpha \ell)^{2}-(\beta \ell)^{2}}{(\alpha \ell)(\beta \ell)} \sinh (\alpha \ell) \sin (\beta \ell)+2\right]=0 \\
& \varphi(0)=0, \quad \varphi^{\prime}(0)=0, \quad \varphi^{\prime \prime}(\ell)+i \omega \eta \varphi^{\prime}(\ell)=0, \quad k^{2} \varphi^{\prime}(\ell)-\varphi^{\prime \prime \prime}(\ell)=0 \\
& \frac{(\alpha \ell)^{4}+(\beta \ell)^{4}}{(\alpha \ell)^{2}(\beta \ell)^{2}} \cosh (\alpha \ell) \cos (\beta \ell)+\frac{(\alpha \ell)^{2}-(\beta \ell)^{2}}{(\alpha \ell)(\beta \ell)} \sinh (\alpha \ell) \sin (\beta \ell)+2 \\
& +i \omega \eta \ell \frac{(\alpha \ell)^{2}+(\beta \ell)^{2}}{(\alpha \ell)^{2}(\beta \ell)^{2}} \cosh (\alpha \ell) \cos (\beta \ell)[\alpha \ell \tanh (\alpha \ell)+\beta \ell \tan (\beta \ell)]=0 \\
& \varphi(0)=0, \quad \varphi^{\prime \prime}(0)-i \omega \eta \varphi^{\prime}(0)=0, \quad \varphi^{\prime \prime}(\ell)+i \omega \nu \eta \varphi^{\prime}(\ell)=0, \quad k^{2} \varphi^{\prime}(\ell)-\varphi^{\prime \prime \prime}(\ell)=0 \\
& \frac{1}{i \omega \eta \ell} \frac{(\alpha \ell)^{2}+(\beta \ell)^{2}}{(\alpha \ell)^{2}(\beta \ell)^{2}}\left[(\alpha \ell)^{3} \tanh (\alpha \ell)-(\beta \ell)^{3} \tan (\beta \ell)\right] \\
& +\frac{1}{\cosh (\alpha \ell) \cos (\beta \ell)}\left[\frac{(\alpha \ell)^{4}+(\beta \ell)^{4}}{(\alpha \ell)^{2}(\beta \ell)^{2}} \cosh \alpha \ell \cos \beta \ell+\frac{(\alpha \ell)^{2}-(\beta \ell)^{2}}{(\alpha \ell)(\beta \ell)} \sinh \alpha \ell \sin \beta \ell+2\right] \\
& +i \omega \nu \eta \ell \frac{(\alpha \ell)^{2}+(\beta \ell)^{2}}{(\alpha \ell)^{2}(\beta \ell)^{2}}[\alpha \ell \tanh (\alpha \ell)+\beta \ell \tan (\beta \ell)]+\nu\left(\frac{(\alpha \ell)^{2}+(\beta \ell)^{2}}{(\alpha \ell)(\beta \ell)}\right)^{2}=0
\end{aligned}
$$



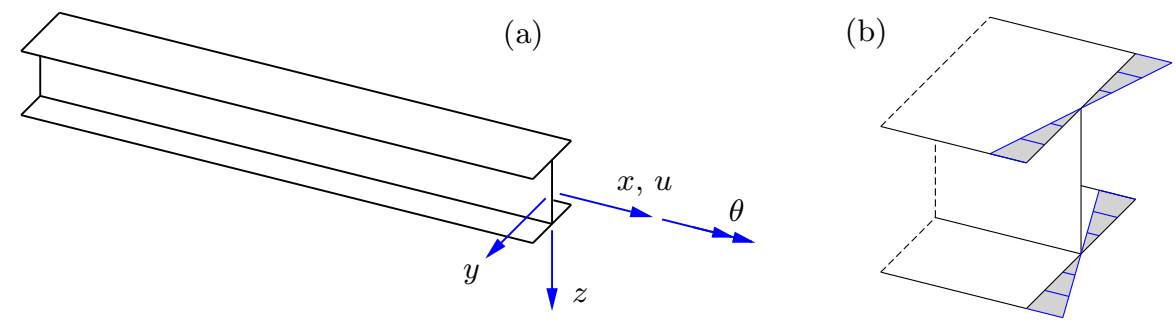

Figure 1. (a) Coordinates and degrees of freedom, (b) warping displacement of beam. 


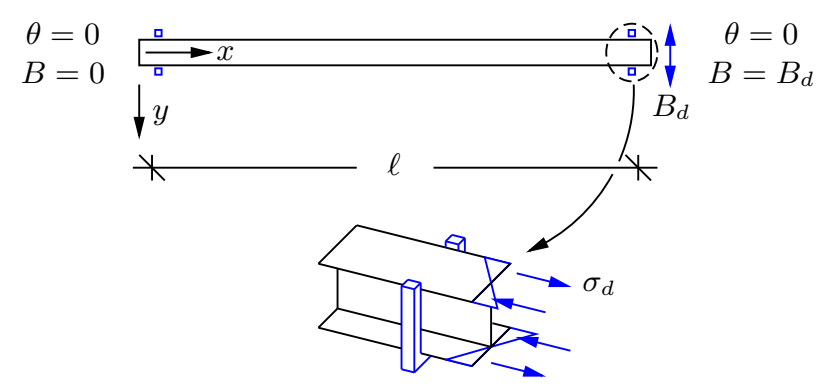

Figure 2. Beam with simple supports and reaction stress $\sigma_{d}$ due to warping displacement at $x=\ell$. 
(I)

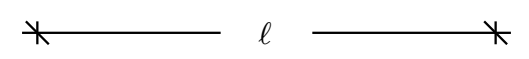

(II)

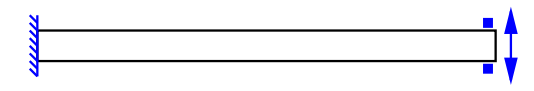

(III)

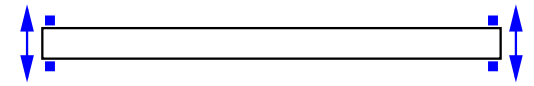

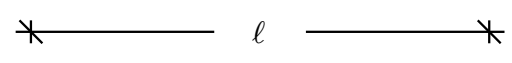

(IV)

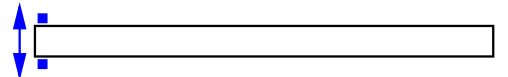

(V)

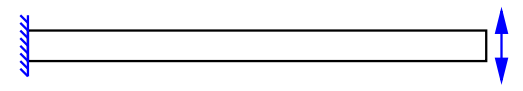

(VI)

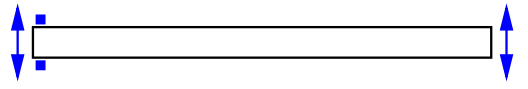

Figure 3. Cases (I)-(VI) with a double arrow representing a viscous bimoment. 


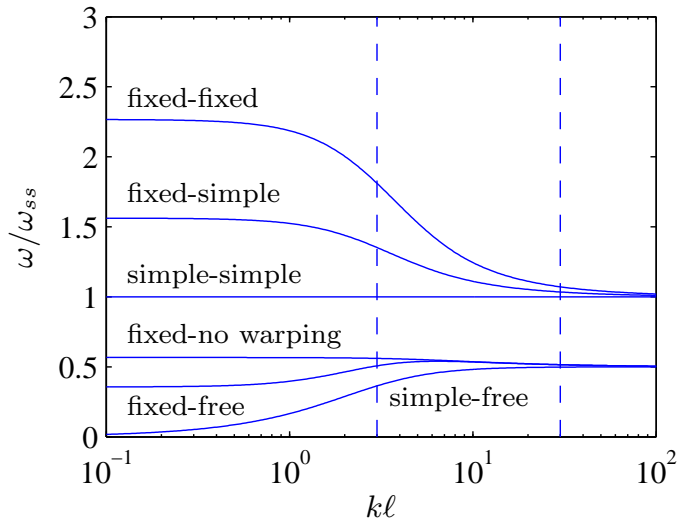

FIgURE 4. Undamped natural frequencies for the first vibration mode. 

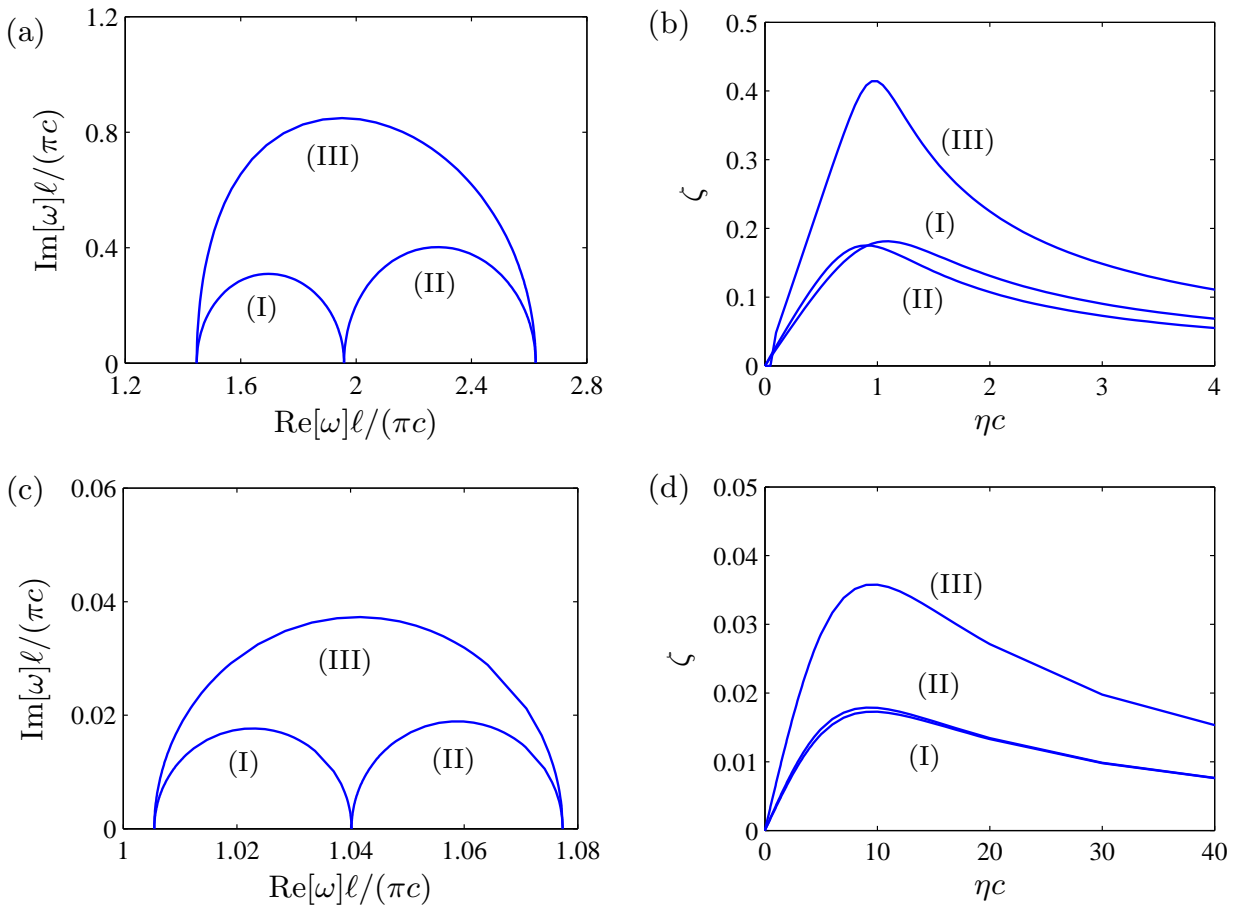

FiguRE 5. Complex frequency loci $(\mathrm{a}, \mathrm{c})$ and damping ratios $(\mathrm{b}, \mathrm{d})$ for $k \ell=3$ $(\mathrm{a}, \mathrm{b})$ and $k \ell=30(\mathrm{c}, \mathrm{d})$. 


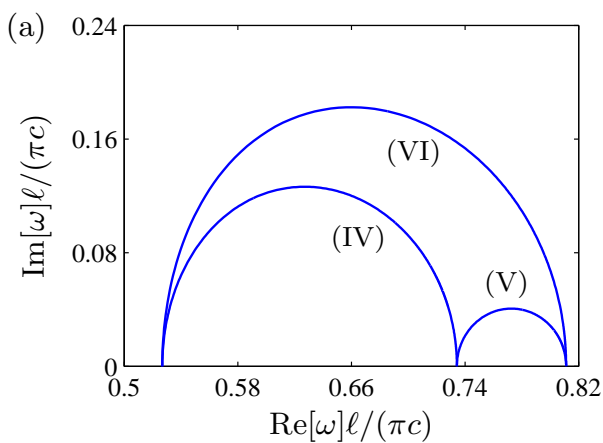

(b)
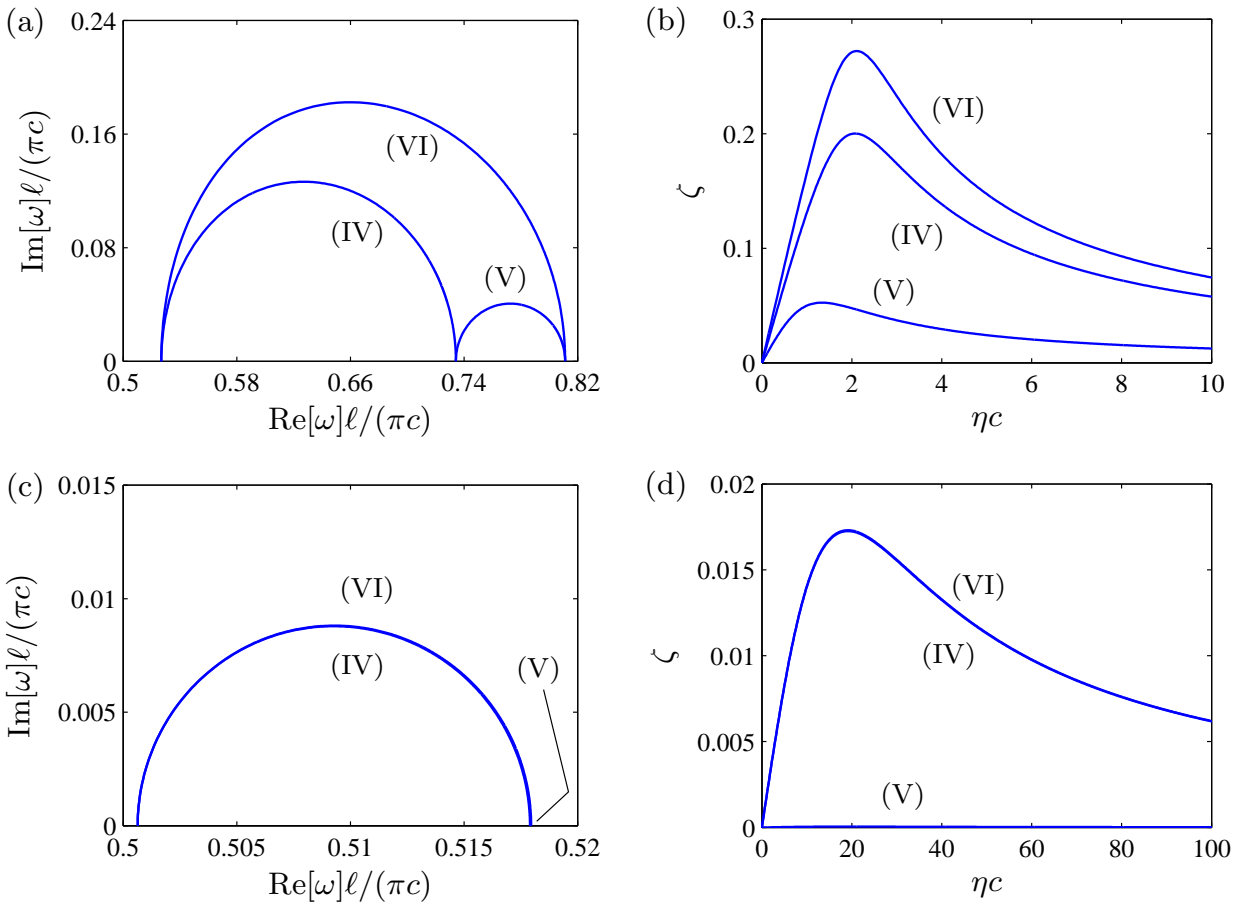

Figure 6. Frequency loci $(\mathrm{a}, \mathrm{c})$ and damping ratios $(\mathrm{b}, \mathrm{d})$ for $k \ell=3(\mathrm{a}, \mathrm{b})$ and $k \ell=30(\mathrm{c}, \mathrm{d})$. 


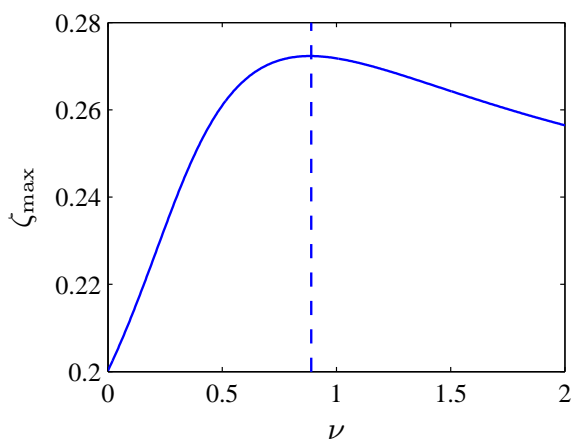

Figure 7. Maximum damping ratio as function of viscous ratio $\nu$ for $k \ell=3$. 